\title{
Precuneus Is a Functional Core of the Default-Mode Network
}

\author{
Amanda V. Utevsky, ${ }^{1,2}$ David V. Smith, ${ }^{3}$ and Scott A. Huettel ${ }^{1,2}$ \\ ${ }^{1}$ Center for Cognitive Neuroscience and ${ }^{2}$ Department of Psychology and Neuroscience, Duke University, Durham, North Carolina 27708, and ${ }^{3}$ Department \\ of Psychology, Rutgers University, Newark, New Jersey 07102
}

Efforts to understand the functional architecture of the brain have consistently identified multiple overlapping large-scale neural networks that are observable across multiple states. Despite the ubiquity of these networks, it remains unclear how regions within these large-scale neural networks interact to orchestrate behavior. Here, we collected functional magnetic resonance imaging data from 188 human subjects who engaged in three cognitive tasks and a resting-state scan. Using multiple tasks and a large sample allowed us to use split-sample validations to test for replication of results. We parceled the task-rest pairs into functional networks using a probabilistic spatial independent components analysis. We examined changes in connectivity between task and rest states using dual-regression analysis, which quantifies voxelwise connectivity estimates for each network of interest while controlling for the influence of signals arising from other networks and artifacts. Our analyses revealed systematic state-dependent functional connectivity in one brain region: the precuneus. Specifically, task performance led to increased connectivity (compared to rest) between the precuneus and the left frontoparietal network (IFPN), whereas rest increased connectivity between the precuneus and the default-mode network (DMN). The absolute magnitude of this effect was greater for DMN, suggesting a heightened specialization for resting-state cognition. All results replicated within the two independent samples. Our results indicate that the precuneus plays a core role not only in DMN, but also more broadly through its engagement under a variety of processing states.

Key words: connectivity; default mode; fMRI; precuneus; resting state

\section{Introduction}

The human brain at rest, when mind-wandering and ruminating, shows distinct and reliable patterns of connectivity among widely separated brain regions. These connectivity networks are thought to reflect intrinsic properties of neural organization (Damoiseaux et al., 2006; Smith et al., 2009). One network that has been of particular interest is the default-mode network (DMN), comprising the posterior cingulate cortex (PCC) and precuneus, medial prefrontal cortex, and bilateral temporoparietal junction. This network of regions gained particular attention when it was shown to decrease in connectivity during tasks-with reduction in connectivity scaling with task difficulty (McKiernan et al., 2003)and increase during rest (Shulman et al., 1997; Raichle et al., 2001; Fox et al., 2005). Building on this foundation, many studies have implicated this network in regulating attentional states and cognition more broadly (Pearson et al., 2011; Leech and Sharp, 2013).

While prior research on relationships between DMN and other networks has primarily adopted antagonistic models, such

Received Sept. 30, 2013; revised Nov. 18, 2013; accepted Dec. 6, 2013.

Author contributions: A.V.U., D.V.S., and S.A.H. designed research; A.V.U., D.V.S., and S.A.H. performed research; A.V.U. and D.V.S. analyzed data; A.V.U., D.V.S., and S.A.H. wrote the paper.

This study was funded by a grant from the National Institutes of Health (NIMH RC1-88680) and an Incubator Award from the Duke Institute for Brain Sciences (S.A.H.). We thank Anne Harsch and Edward McLaurin for assistance with data collection, and R. McKell Carter, John Clithero, Vinod Venkatraman, Amy Bland, and Sarah Boltuck for their aid in data collection and study design.

Correspondence should be addressed to Scott A. Huettel, Box 90999, Duke University, Durham, NC 27708. E-mail: scott.huettel@duke.edu.

DOI:10.1523/JNEUROSCI.4227-13.2014

Copyright $\odot 2014$ the authors $\quad 0270-6474 / 14 / 340932-09 \$ 15.00 / 0$ that DMN engagement is temporally anticorrelated with taskrelated networks (Fox et al., 2005; Fransson, 2005), recent research suggests that such models may be overly simplistic. Although the PCC/precuneus is thought to comprise the functional core of $\mathrm{DMN}$, the region exhibits connectivity patterns that differ from the larger network: as task difficulty increases, ventral and dorsal PCC/precuneus exhibit opposite patterns of integration with DMN; further, while at rest, dorsal PCC/precuneus shows connectivity with both DMN and task-positive networks (Leech et al., 2011). Other studies have shown that PCC/precuneus exhibits increased activation during many tasks-including autobiographical memory retrieval (Maddock et al., 2001), reward outcome monitoring (Hayden et al., 2008), and emotional stimulus processing (Maddock et al., 2003)-further challenging the association of DMN with task disengagement, and highlighting the differences between the functional core of DMN and the network more broadly. Understanding the role of DMN and its relationship with task-related networks during both resting and task states may resolve these contrasting findings and provide critical insight into the network's involvement in regulating cognition.

We investigated the relationship between DMN and taskpositive networks by comparing connectivity during resting and task states. We collected functional neuroimaging data from 188 subjects who engaged in three cognitive tasks in separate scans and a resting-state scan. Data from each task were paired with corresponding resting-state data, split into primary and replication samples, and parceled into functional networks using spatial independent components analysis (Beckmann et al., 2005; Smith et al., 2009). We examined connectivity during task and rest using 


\section{Identifying State-Dependent Network Coactivation}

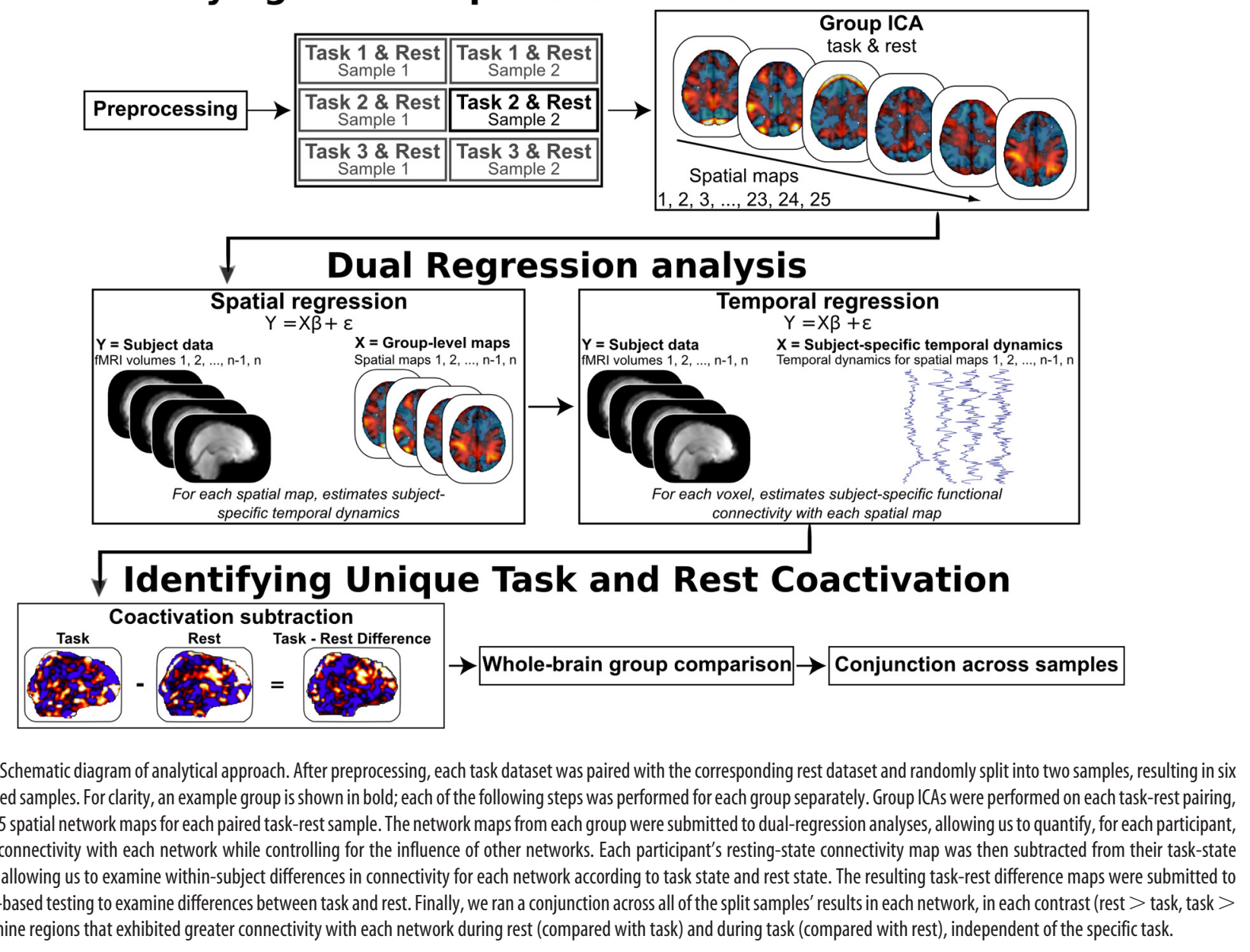

Figure 1. Schematic diagram of analytical approach. After preprocessing, each task dataset was paired with the corresponding rest dataset and randomly split into two samples, resulting in six task-rest paired samples. For clarity, an example group is shown in bold; each of the following steps was performed for each group separately. Group ICAs were performed on each task-rest pairing, resulting in 25 spatial network maps for each paired task-rest sample. The network maps from each group were submitted to dual-regression analyses, allowing us to quantify, for each participant, each voxel's connectivity with each network while controlling for the influence of other networks. Each participant's resting-state connectivity map was then subtracted from their task-state connectivity, allowing us to examine within-subject differences in connectivity for each network according to task state and rest state. The resulting task-rest difference maps were submitted to permutation-based testing to examine differences between task and rest. Finally, we ran a conjunction across all of the split samples' results in each network, in each contrast (rest $>$ task, task $>$ rest), to examine regions that exhibited greater connectivity with each network during rest (compared with task) and during task (compared with rest), independent of the specific task.

dual-regression analysis (Fig. 1), which quantifies voxelwise connectivity estimates for each network of interest, while controlling for the influence of other networks (Filippini et al., 2009; Leech et al., 2011; Sharp et al., 2011). Our results indicate that precuneus exhibits heightened connectivity with both DMN and a taskpositive network according to task state, and additionally exhibits connectivity patterns that reliably discriminate task state. This observation indicates that precuneus plays a core role not only in DMN, but also cognition more broadly through its engagement under a variety of processing states.

\section{Materials and Methods}

Participants and experimental tasks. A group of 209 participants completed three tasks and a resting-state scan. During the resting-state scan, participants were given instructions to maintain fixation on a central cross and to not think about anything particular. Before the resting-state scan, participants engaged in three reward-based decision tasks requiring externally focused attention. All tasks share a common structure, with periods of rest (i.e., fixation) embedded within periods of active decision making, evaluation, and response selection. Although the precise timing of each task is not central to the core analyses-which compare task state and rest state-we here include a brief description of each task.

First, participants engaged in a variant of a popular incentivized response-time task (Knutson et al., 2000; Clithero et al., 2011). On each trial, subjects were presented with a $1 \mathrm{~s}$ cue, indicating whether a sufficiently fast response would earn a monetary reward or nothing. Subjects were then presented with a fixation cross, jittered between 1.75 and $2.75 \mathrm{~s}$, and then a $0.1-0.3 \mathrm{~s}$ cue, prompting them to respond. The subjects' task was to respond by button press before the cue disappeared. The response period was then followed by an intertrial interval (ITI) between 2 and $8 \mathrm{~s}$.

Following the incentivized response-time task, participants completed a financial decision-making task that has been used previously to study framing effects (De Martino et al., 2006). On each trial, participants saw a cue for $1 \mathrm{~s}$ that indicated an initial monetary endowment, as well as whether the monetary outcome of the following decision would be donated to the subject or to the subject's chosen charity. Following the endowment cue, subjects had a $3.5 \mathrm{~s}$ decision period in which they had to choose between a "sure" option (keep or lose the entire endowment) or a "gamble" option that indicated the probability of keeping or losing the endowment. The decision period was then followed by an ITI, jittered between 2.5 and $6.5 \mathrm{~s}$.

After the financial decision-making task, participants completed a simple valuation task aimed at examining representation of risk in the brain. This task comprised gambles whose earnings varied in magnitude and probability (adapted from Huettel et al., 2006). On each trial, subjects first saw a cue, jittered between 2.25 and $6.3 \mathrm{~s}$, indicating either the probabilities of monetary gain and loss or the magnitudes of each possible outcome. Following this initial cue, subjects were presented with the remaining information (e.g., if probability had been shown first, they then saw magnitude) for 4-10 s. Once provided with complete information about the gamble, subjects were presented with a valuation screen for 2.25-6.3 s. After expressing a value for the gamble, the outcome was revealed for $1 \mathrm{~s}$, and then followed by an ITI, jittered between 2.5 and $7.5 \mathrm{~s}$

Individuals with prior psychiatric or neurological illness were excluded; other individuals were excluded based on data quality concerns (see below, Preprocessing), or nonresponsive behavior on tasks. These exclusion criteria left a final sample of 188 total subjects ( 85 males, $103 \mathrm{fe}-$ 
males; ages: $18-59$, mean $=22$; level of education: $12-24$, mean $=15$; Experiment 1 dataset: $\mathrm{N}=177$; Experiment 2 dataset: $\mathrm{N}=179$; $\operatorname{Exp} 3$ dataset: $\mathrm{N}=183$; each of the three tasks comprised different subsets of subjects, depending on quality assurance measures). Due to the large sample sizes, we chose to randomly split each task-rest sample into primary and replication sets to explicitly test for replication of findings (Experiment 1 dataset 1: $\mathrm{N} 1=88$; Experiment 1 dataset 2: $\mathrm{N} 2=89$; Experiment 2 dataset 1 : $\mathrm{N} 1=90$; Experiment 2 dataset 2: $\mathrm{N} 2=89$; Experiment 3 dataset 1: $\mathrm{N} 1=92$; Experiment 3 dataset 2: $\mathrm{N} 2=91$ ). All participants gave written informed consent as part of a protocol approved by the Institutional Review Board of Duke University Medical Center.

Image acquisition. Scanning was performed using a General Electric MR750 3.0 T scanner using an 8-channel parallel imaging system. $\mathrm{T}_{2}{ }^{*}$ weighted images were acquired using a spiral-in sensitivity encoding sequence (acceleration factor $=2$ ) with slices parallel to the axial plane connecting the anterior and posterior commissures [repetition time (TR): $1580 \mathrm{~ms}$; echo time (TE): $30 \mathrm{~ms}$; matrix: $64 \times 64$; field of view (FOV): $243 \mathrm{~mm}$; voxel size: $3.8 \times 3.8 \times 3.8 \mathrm{~mm}$; 37 axial slices; flip angle: 70 degrees]. This sequence was chosen to ameliorate susceptibility artifacts (Pruessmann et al., 2001; Truong and Song, 2008), particularly in the ventral frontal regions often identified within the DMN (Gusnard and Raichle, 2001; Fox et al., 2005; Fox and Raichle, 2007). Before preprocessing these functional data, the first eight volumes of each run were removed to allow for magnetic stabilization. All functional datasets were truncated at 192 volumes to facilitate comparisons with the resting-state scan. Whole-brain high-resolution anatomical scans were acquired $\left(\mathrm{T}_{1^{-}}\right.$ weighted FSPGR sequence; TR: $7.58 \mathrm{~ms}$; TE: $2.93 \mathrm{~ms}$; matrix: $256 \times 256$; FOV: $256 \mathrm{~mm}$; voxel size: $1 \times 1 \times 1 \mathrm{~mm}$; 206 axial slices; flip angle: 12 degrees) to facilitate coregistration and normalization of functional data.

Preprocessing. Our preprocessing routines used tools from the FMRIB Software Library (FSL Version 4.1.8; http://www.fmrib.ox.ac.uk/fsl/) package (Smith et al., 2004; Woolrich et al., 2009). We corrected for head motion by realigning the time series to the middle volume (Jenkinson and Smith, 2001), and then removed nonbrain material using a brain extraction tool (Smith, 2002). Next, we corrected intravolume slicetiming differences using Fourier-space phase shifting, aligning to the middle slice (Sladky et al., 2011). Images were then spatially smoothed using a $6 \mathrm{~mm}$ full-width-half-maximum Gaussian kernel. Next, we used a liberal high-pass temporal filter with a 150 s cutoff (Gaussian-weighted least-squares straight line fitting, with $\sigma=75 \mathrm{~s}$ ), because of the broadband spectral power observed in resting-state fluctuations (Niazy et al., 2011). Each 4 D dataset was then grand-mean intensity normalized using a single multiplicative factor. Finally, we spatially normalized functional data to the Montreal Neurological Institute (MNI) avg152 T1-weighted template ( $3 \mathrm{~mm}$ isotropic resolution) using a 12-parameter affine transformation implemented in FLIRT (Jenkinson and Smith, 2001).

As part of our preprocessing steps and quality control, we examined three partially correlated measures of quality assurance and excluded subjects who exhibited extreme values on these metrics. First, we estimated the average signal-to-fluctuation-noise ratio (SFNR) for each subject (Friedman and Glover, 2006). Second, we computed the average volume-to-volume motion for each subject. Third, we identified outlier volumes in our functional data by evaluating the root-mean-square error (RMSE) of each volume relative to the reference volume (the middle time point); a volume was considered an outlier if its RMSE amplitude exceeded the 75 th percentile plus the value of $150 \%$ of the interquartile range of RMSE for all volumes in a run (i.e., a standard boxplot threshold). After calculating these metrics, we excluded subjects where any measure was extreme relative to the other subjects (i.e., SFNR $<5$ th percentile of the distribution of SFNR values; outlier volumes $>$ 95th percentile the distribution of outlier volumes; average volume-tovolume motion $>$ 95th percentile).

Although excluding outliers in connectivity analyses is crucial, as artifacts (e.g., motion) can severely distort results (Jansen et al., 2012; Power et al., 2012; Satterthwaite et al., 2012), it is equally important to address data quality concerns in the remaining subjects. To do so, we regressed out variance tied to six parameters describing motion (rotations and translations along the three principal axes), as well as volumes identified as outliers. While we did not use the scrubbing procedure described by
Power et al (2012), our method of regressing out outlier volumes accomplishes the same goal of removing signal discontinuities and nonlinear effects of head movement that cannot be accounted for by conventional motion parameters or derivations of motion parameters.

Independent components analyses. We submitted each of the six groups' data (three task-rest pairs, each split into primary and replication groups) to separate probabilistic group independent components analysis (ICA; Beckmann and Smith, 2004), as used in FSL's Multivariate Exploratory Linear Decomposition into Independent Components (MELODIC) Version 3.10. ICAs are able to identify coherent neural networks by extracting structured signals that exist simultaneously in data; additionally, ICAs can separate key signals of interest (i.e., patterns of coactivation) from artifactual or physiological noise and head motion.

The data submitted to each of the six group ICAs comprised both resting-state data and task data, such that each dataset had two inputs for each subject: one resting-state scan and one task-based scan. Before ICA estimation, the data were further processed using voxelwise de-meaning and by normalizing the voxelwise variance. The processed data were whitened and then projected into a 25 -dimensional subspace. We selected 25 components based on prior reports examining multiple restingstate networks (RSNsFilippini et al., 2009; Sharp et al., 2011); we note, however, that the number of components primarily affects the granularity of the estimated networks (Smith et al., 2009). The whitened data were then decomposed into sets of vectors describing the signal variation across (1) time (time courses) and (2) space (component maps), using a fixed-point iteration technique (Hyvärinen, 1999) to optimize nonGaussian spatial source distributions. The estimated network maps were thresholded by dividing each map by the SD of the residual noise, and then fitting a Gaussian mixture model to the histogram of normalized intensity values (Beckmann and Smith, 2004).

Dual-regression analyses. To examine changes in connectivity between task and rest, we submitted network maps from each group to a separate dual-regression analysis, which quantifies voxelwise connectivity estimates for each network, while controlling for the influence of other networks (Filippini et al., 2009; Leech et al., 2011, 2012). Each dualregression analysis comprised two stages (Fig. 1). First, each network map was regressed onto each subject's individual functional dataset, resulting in subject-specific time courses for each network. Second, these resulting time courses were then regressed onto the subject's functional data to estimate, within each subject, each voxel's connectivity with each independent network. Importantly, the second, temporal regression examines each voxel's connectivity with each spatial network while controlling for any influence of other networks, including those that are potentially artifactual. To quantify task-rest connectivity changes within each subject, each participant's resting-state connectivity map was subtracted from their task-state connectivity map, resulting in difference images indicating task-minus-rest changes in connectivity.

Using these subject- and network-specific connectivity difference maps, we then constructed a group-level general linear model to estimate changes in each network according to task state and rest state. Importantly, we modeled subject-level estimates of motion to account for any consistent differences due to the participant performing a task or being at rest. Specifically, we included two nuisance regressors that summarized individual differences in motion (average volume-to-volume motion and the proportion of outlier volumes identified), as well as a regressor indicating SFNR, as differences in SFNR may be associated with spurious differences in connectivity (Friston, 2011; O'Reilly et al., 2012). These regressors were calculated by subtracting the parameter values in the resting-state scan from those in the task-state scan, providing a taskminus-rest difference value for each metric and further controlling for any spurious differences that could arise due to subjects moving more while performing a task compared with rest. Additionally, we included a regressor to account for sex differences (Filippi et al., 2013), as well as a dummy covariate to account for a subtle change in scanning parameters that began partway through data collection (i.e., the utilization of a fat saturation pulse).

To identify RSNs from our ICA that corresponded to the four networks of interest, we conducted a spatial correlation analysis for each 
A
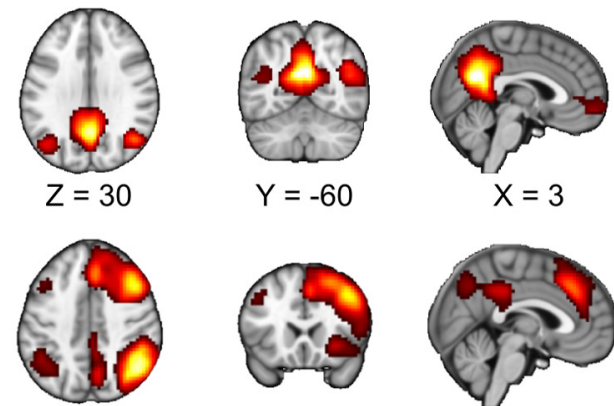

$\mathrm{Y}=-60$

$\mathrm{X}=3$

$Z=42$

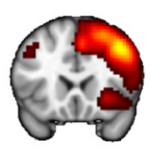

$Y=21$

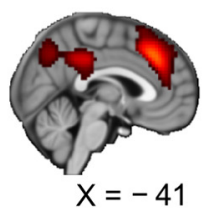

B

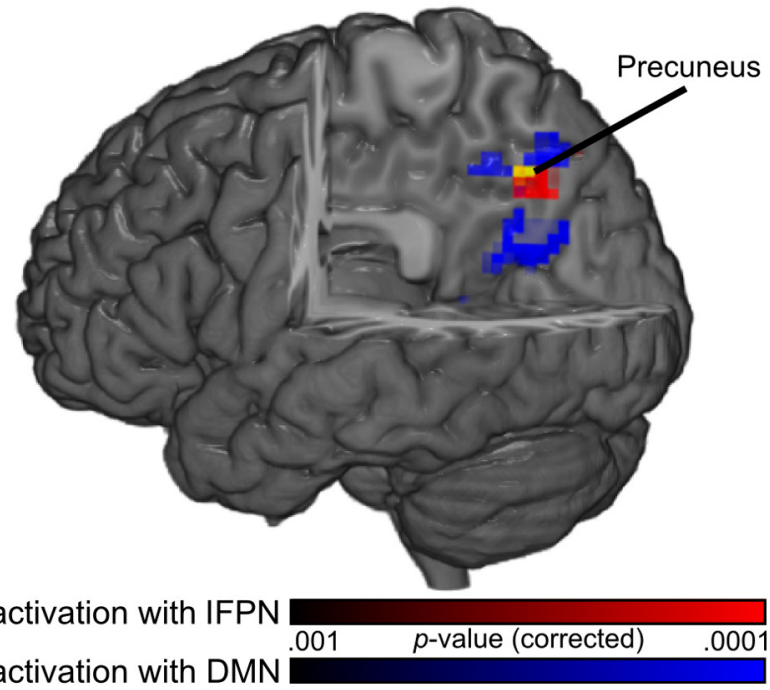

Figure 2. Overlap in connectivity with DMN and frontoparietal network. $A$, We identified two networks that exhibit significant connectivity differences in the task and rest states, the default-mode network (top), and the left frontoparietal network (bottom). Activations shown are the intersections of each $z$-transformed network map, respectively, across the six groups examined. $\boldsymbol{B}$, Areas within precuneus exhibited task-dependent connectivity with IFPN (red) and DMN (blue). Strikingly, a subregion in the precuneus (coordinates: 6, -63, 42; yellow) differentiated task states in both networks, indicating that this region is a shared node between multiple networks.

Table 1. State differences in connectivity with DMN

Rest $>$ task $(p<0.00625$, whole-brain corrected)

\begin{tabular}{|c|c|c|c|c|c|}
\hline Probabilistic anatomical label & $x$ & $y$ & $z$ & $p$ value & Cluster extent \\
\hline $\begin{array}{l}\text { Supracalcarine }(31 \%) \text {, precuneus }(20 \%) \text {, } \\
\text { cuneus }(14 \%) \text {, intracalcarine }(6 \%)\end{array}$ & 15 & -63 & 18 & 0.001 & 116 \\
\hline Precuneus (65\%) & 0 & -54 & 45 & 0.001 & 58 \\
\hline $\begin{array}{l}\text { Cuneus }(20 \%) \text {, supracalcarine }(16 \%) \text {, } \\
\text { precuneus }(15 \%) \text {, intracalcarine (13\%) }\end{array}$ & -12 & -69 & 18 & 0.001 & 55 \\
\hline Cingulate gyrus (17\%) & 6 & -42 & 3 & 0.008 & 2 \\
\hline Cingulate $(15 \%)$ & -3 & -45 & 6 & 0.007 & 1 \\
\hline
\end{tabular}

Regions whose connectivity with the DMN was greater in the resting state compared to the task state, after correcting for two-tailed tests across the four independent networks. Regions were found through a conjunction analysis across the six task-rest pairings (see Materials and Methods). Probabilistic labels refer to the likelihood that the coordinate exists within the given cortical region. In cases where multiple labels are ascribed to a single coordinate, we only show labels whose likelihood exceeds $5 \%$.

network from the ICAs and each of the four networks selected from Smith et al. (2009). Within each of the six datasets, we selected the maps that best matched each of the three task-positive (executive control, left and right frontoparietal networks; rFPN) and one task-negative network (DMN) of interest in Smith et al. (2009). We performed bidirectional contrasts comparing task and rest states in each of the four RSNs. Statistical significance was assessed in a nonparametric fashion, using Monte Carlo permutation-based statistical testing with 10,000 permutations with $\alpha=0.05$ corrected for multiple comparisons across the whole brain
Table 2. State differences in connectivity with left frontoparietal network

\begin{tabular}{llllll}
\hline Task $>$ rest $(p<0.00625$, whole-brain corrected) & & & \\
\hline Probabilistic anatomical label & $x$ & $y$ & $z$ & $p$ value & Cluster extent \\
\hline Precuneus (58\%) & 6 & -63 & 39 & 0.001 & 21 \\
Precuneus (32\%), cuneus (22\%), & 9 & -75 & 45 & 0.009 & 1 \\
$\quad$ lateral occipital (13\%) & & & & &
\end{tabular}

Regions whose connectivity with the IFPN was greater in the task state compared to the resting state, after correcting for two-tailed tests across the four independent networks. Regions were found through a conjunction analysis across the six task-rest pairings (see Materials and Methods). Probabilistic labels refer to the likelihood that the coordinate exists within the given cortical region. In cases where multiple labels are ascribed to a single coordinate we only show labels whose likelihood exceeds $5 \%$.

as well as the four networks tested (Nichols and Holmes, 2002). To estimate clusters of activation, we used threshold-free cluster enhancement (Smith and Nichols, 2009). Brain activations are displayed using MRIcroGL (http://www.mccauslandcenter.sc.edu/mricrogl/).

To identify brain regions that show state-based connectivity differences independent of any specific task, we conducted conjunction analyses across all six groups' connectivity maps along both contrasts (task $>$ rest, rest $>$ task) using the minimum statistic (Nichols et al., 2005). All coordinates reported are in MNI space.

\section{Results}

Functional connectivity networks are modulated by task state Our initial analyses compared voxelwise connectivity estimates with multiple networks at rest and task. We predicted that regions within DMN would exhibit increased connectivity with the network at rest compared with task, consistent with prior reports of elevated activation of DMN during rest (Greicius et al., 2003). In contrast, we hypothesized that regions within task-based networks would show increased connectivity with those networks during task compared with rest. To test these hypotheses, and, crucially, to ensure consistent results across multiple independent samples, we conducted a conjunction analysis across all six groups for each of the three task-positive networks-the salience network, the left and right frontoparietal networks-and the tasknegative DMN (Fox et al., 2005). Of these four networks, the left frontoparietal network (1FPN; Fig. 2A) and default-mode network (DMN; Fig. 2A) exhibited replicable differences in connectivity according to task state. Conjunction analyses across the DMN connectivity maps revealed greater connectivity with the network at rest compared with task; these differences were found in the supracalcarine cortex, intracalcarine cortex, precuneal cortex, cuneal cortex, and cingulate cortex (Table 1). In contrast, the conjunction analysis across the IFPN connectivity maps revealed significant connectivity with the network in the opposite direction-heightened at task compared to rest; this difference was found in precuneal and cuneal cortex (Table 2). The separate conjunctions across the DMN and IFPN connectivity maps revealed one region of overlap within the precuneus (coordinates: $6,-63,42$; Fig. $2 B$ ); this region showed state-dependent connectivity with both networks in opposite contrasts.

\section{Precuneus is a distinct hub within the DMN}

To investigate whether the overlap region observed in precuneus exhibited network-specific connectivity, we compared the magnitude of connectivity with DMN and lFPN. We found that the absolute magnitude of connectivity of the precuneus subregion was greater with the DMN than with the lFPN in both the primary and replication groups (collapsed across task; $p<0.0001$ both sets; Fig. $3 A$, rightmost sets of bars), indicating heightened integration of the precuneus during the resting state.

We also examined whether the pattern of connectivity we found was specific to this subregion of the precuneus, or applied 


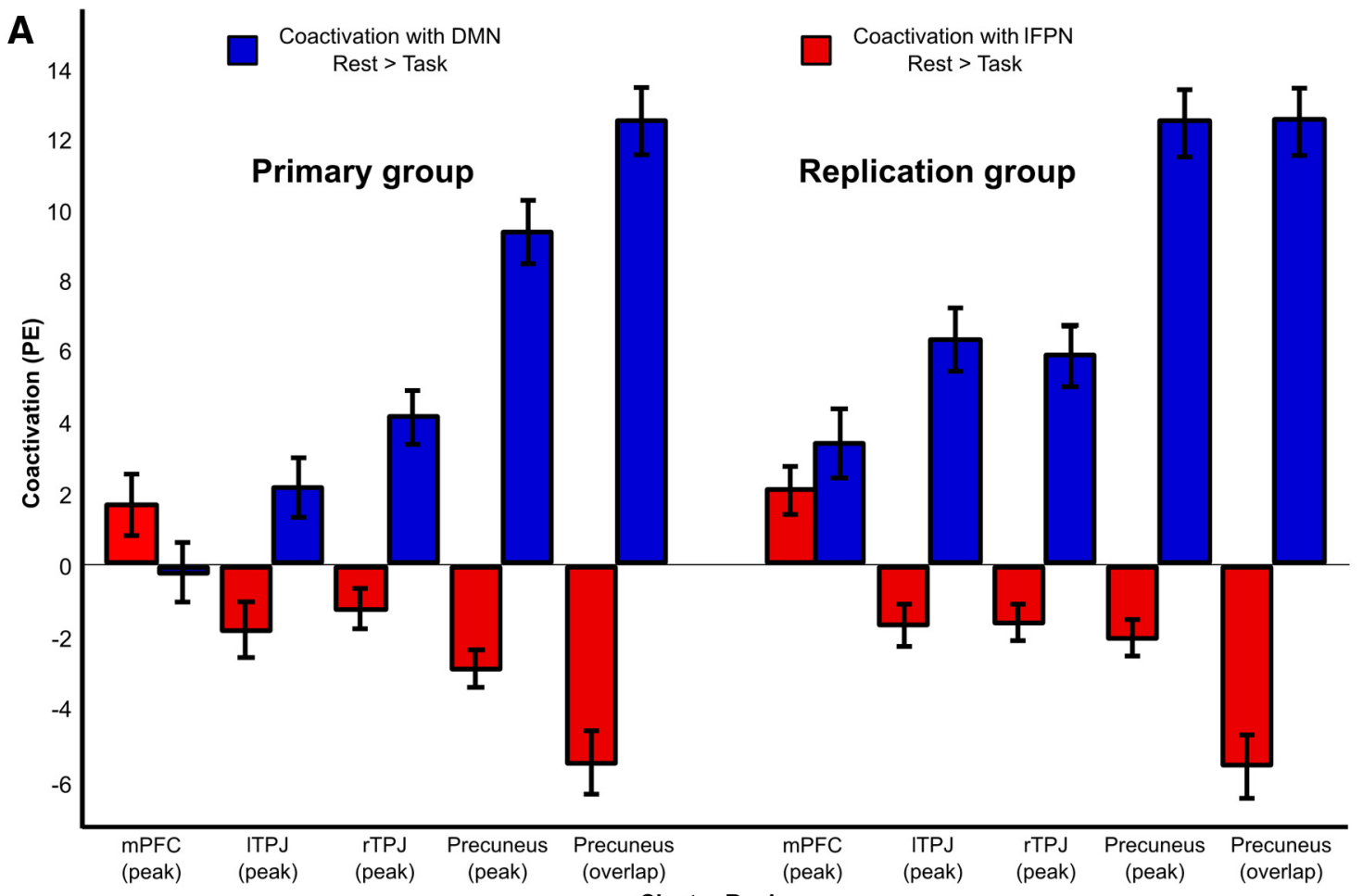

B
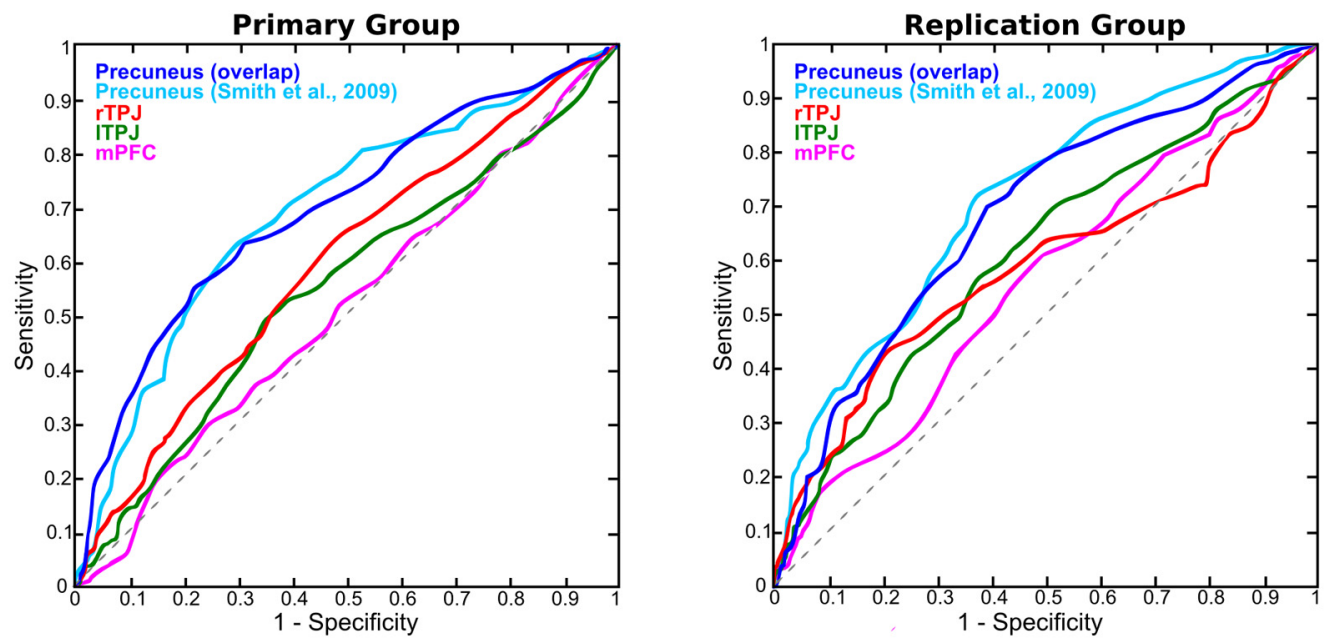

Figure 3. Connectivity between DMN at rest and typical DMN regions. We examined connectivity between peak voxels of canonical default-mode network (DMN) regions (Smith et al., 2009) and our rest $>$ task and task $>$ rest connectivity maps for both the DMN and the left frontoparietal (IFPN) networks. A, Parameter estimates quantifying subject-specific rest-minus-task connectivity between the selected region and the DMN (blue) and IFPN (red) in both the primary groups of each task and the replication groups of each task. Error bars indicate SEM across subjects. In both the primary and replication samples of the three tasks, the precuneal region identified by the overlap of connectivity exhibited significantly greater connectivity with the DMN at rest (compared with task) than any other region tested. This pattern replicated in a spatially distinct region of the precuneus that was chosen from an independent DMN map (Smith et al., 2009). $\boldsymbol{B}$, To examine the role of the precuneus within the DMN, ROCs were computed for our precuneal region and the peak voxel of canonical DMN regions (Smith et al., 2009). In both our primary and replication groups of the three task-rest parings, we found that rest-minus-task connectivity estimates between our precuneal overlap region and DMN were significantly better at distinguishing task state and rest state than any of the other typical DMN regions tested (Table 3). This result was replicated in a spatially separate precuneal region, selected from an independent DMN map (Smith et al., 2009). rTPJ, right temporoparietal junction; ITPJ, left temporoparietal junction; mPFC, medial prefrontal cortex.

to the precuneus more broadly. We therefore selected the peak voxel from the precuneal region of activation in an independent DMN map (coordinates: 3, -60, 30; Smith et al., 2009). We found that this spatially distinct cluster exhibited a similar pattern of connectivity as the precuneal subregion found from our conjunction analysis (Fig. 3A); specifically, this distinct precuneus region exhibited heightened connectivity with DMN compared with IFPN in both the primary and replication groups (collapsed across task; $p<0.0001$ for both sets).
Although our results highlight the importance of the precuneus within the DMN, it remains unclear whether this region serves a specialized function relative to other DMN regions. To examine this possibility, we compared our precuneus subregion to other regions typical of the DMN. Specifically, we created 5 $\mathrm{mm}$ spheres around each peak voxel of DMN network activation from an independent activation map (Smith et al., 2009), and compared the regions' connectivity with our DMN map. These regions included the precuneus (coordinates: $3,-60,30$ ), the 
Table 3. Precuneus distinctively distinguishes task and rest states

\begin{tabular}{llc}
\hline Region & AUC & $p$ value \\
\hline Precuneus (overlap) & 0.69 & - \\
Precuneus & 0.69 & 0.518 \\
mPFC & 0.51 & $<0.001$ \\
ITPJ & 0.55 & $<0.001$ \\
rTPJ & 0.59 & 0.006 \\
& & - \\
Precuneus (overlap) & 0.69 & 0.716 \\
Precuneus & 0.71 & $<0.001$ \\
mPFC & 0.556 & 0.02 \\
ITPJ & 0.61 & 0.003 \\
rTPJ & 0.59 & \\
\hline
\end{tabular}

ROCs were computed for each region of activation in an independent DMN map (Smith et al., 2009; Figure 3B) and our precuneal region of overlap. AUCs are listed for each region examined. We compared AUCs from each independently selected region to the AUC of our precuneal region using permutation-based testing $(10,000$ permutations); $p$ values indicate differences between the region and our precuneal region of overlap. The blank row separates the connectivity estimates for the DMN map from the primary sample (above) and the replication sample (below). ITPJ, left temporoparietal junction; mPFC, medial prefrontal cortex; rTPJ, right temporoparietal junction.

paracingulate gyrus (coordinates: $0,54,-3$ ), the left lateral occipital cortex (coordinates: $-48,-66,36)$, and right lateral occipital cortex (coordinates: 54, $-60,27$ ). Strikingly, among the various DMN-typical regions tested, our precuneus subregion showed significantly greater connectivity with the DMN at rest compared with task than any of the other regions tested; this was found in both the primary and replication groups (Fig. $3 A$ ). Importantly, this pattern of results was similar when evaluating the independent, spatially separable precuneus region (Smith et al., 2009), suggesting that the precuneus more broadly serves a distinct function relative to the other regions in the network.

To further evaluate whether the precuneus serves a specialized function within the DMN, we examined the receiver-operating characteristic (ROC) curves for each of these regions. We compared the area under the curves (AUC) for the other DMN regions examined previously (Fig. $3 B$ ). Of the four regions tested, our precuneus region exhibited significantly greater AUC than the three other regions in both the primary and replication samples (Table 3). Again, this pattern of results was also true for the precuneus region selected from an independent DMN map (Smith et al., 2009). These results corroborate the earlier evidence suggesting a distinctive role of the precuneus in the DMN, as they indicate that the connectivity between precuneus and DMN distinguishes whether subjects are at task or at rest better than any of the other canonical DMN regions.

\section{Discussion}

Large-scale neural networks, particularly the DMN (Raichle et al., 2001; Beckmann et al., 2005), have emerged as a focal point in a diverse set of neuroscience studies, from neuroimaging in humans (Greicius and Menon, 2004; Fox et al., 2006; Buckner and Carroll, 2007) to single-unit recordings in animals (Hayden et al., 2009, 2010).

Yet, how different large-scale networks interact to orchestrate task- and rest-state behavior has remained elusive. We investigated this issue and found that the precuneus exhibits statedependent interactions with both the left-lateralized frontoparietal network (IFPN) and the default-mode network (DMN). Moreover, the precuneus showed a distinct pattern of connectivity with the DMN, suggesting it may be a specialized nexus within the network.

Considerable prior neuroimaging has implicated the precuneus as a central node in the human brain, important for supporting complex cognition and behavior. The precuneus comprises a core region of the DMN, exhibiting decreased activation during most externally driven tasks (Raichle et al., 2001; Fransson, 2005), and reliable increases in activation in response to both rest and specific tasks, such as autobiographical memory (Addis et al., 2004), as well as unique interactions with the rest of the network (Fransson and Marrelec, 2008). Its role in the DMN has been of particular interest as it shows the highest resting metabolic rate within the network, requiring $\sim 35 \%$ more glucose than any other region in the human brain (Gusnard and Raichle, 2001). Further, the widespread connectivity of the precuneus, involving higher association regions, suggests an important role in integrating both internally and externally driven information (Cavanna and Trimble, 2006). Still, the function of the region is unknown. While the precuneus shows heightened activation during episodic (Fletcher et al., 1995; Lundstrom et al., 2005) and autobiographical memory (Addis et al., 2004; Eustache et al., 2004) tasks, it similarly shows heightened activation during rest (Raichle et al., 2001), an effect that is augmented in single units during increased disengagement from the task being performed (Hayden et al., 2009). Our results extend a unifying function of these competing results (Leech et al., 2011) by acknowledging that the precuneus is functionally variable, exhibiting connectivity with different neural networks according to task state or level of engagement with one's surroundings and demonstrating that connectivity between the precuneus and the DMN reflects this level of engagement.

We used a relatively novel analysis scheme in this study to compare connectivity with networks in different cognitive states. Our analyses allowed us three important advantages over previous research examining task-dependent connectivity changes (Fransson and Marrelec, 2008; Mennes et al., 2010, 2013; Gordon et al., 2012). First, dual-regression techniques allowed us the ability to investigate connectivity with multiple distinct networks while controlling for the influence of other, potentially overlapping, networks (Filippini et al., 2009; Leech et al., 2012). This is crucial, as typical seed-based analyses would have been unable to distinguish the separate responses from DMN and IFPN in our study, whereas ICA and dual-regression were able to parse connectivity of spatially overlapping networks (Cole et al., 2010). Second, having such a large sample size allowed us to verify that the results from our dual-regression analyses held in an independent sample, thus attenuating the odds that our observations are due to Type 1 errors (Button et al., 2013). Finally, comparing the resting state to three distinct tasks allowed us the ability to identify differences in connectivity that exist independently of the task and can generalize more broadly, which has not been feasible in previous studies (Fransson and Marrelec, 2008; Gordon et al., 2012).

We note that our analytical procedure also represents a departure from other studies examining hubs of functional connectivity with variants of graph-theoretic approaches (Sporns et al., 2007; Buckner et al., 2009). While our approach identified precuneus as a hub by examining the interplay of multiple interacting networks under distinct processing states, graph-theoretical approaches generally seek to identify hubs by quantifying the strength of connectivity between nodes within a single network at rest (for review, see Bullmore and Sporns, 2009). Such approaches can be powerful, but they can introduce problems if their nodes do not correspond well to functional boundaries (e.g., if regions are drawn from anatomical atlases); that is, inappropriate node definitions can limit subsequent graph analyses (Smith, 2012), potentially distorting claims regarding hubs. Recent work has avoided this pitfall by focusing on voxelwise met- 
rics of resting-state functional connectivity density, implicating the precuneus as a key cortical hub (Tomasi and Volkow, 2010). We speculate that combining our approach with these earlier approaches could integrate the advantages of both methods, revealing additional cortical hubs that contribute to a range of processing states.

A potential limitation of our study is that the three tasks examined involved reward-related decision making. While the tasks all differ from one another in many ways, common taskbased connectivity may have arisen due to potentially common processes. For example, a recent study demonstrated distinct connectivity with the DMN under different memory processes (Shapira-Lichter et al., 2013), suggesting that different cognitive demands of a task may give rise to different patterns of connectivity. While our study demonstrated that the precuneus exhibits task-dependent connectivity with IFPN and DMN when examining three reward-based decision-making tasks, it is possible that other regions in the networks, such as intraparietal or medial prefrontal cortex, may exhibit similar behavior under different task conditions, such as autobiographical memory or social cognition tasks. We note that our finding that precuneus exhibits changes in connectivity is consistent with prior research examining task-rest changes using a working memory task (Fransson and Marrelec, 2008), suggesting that our results are not specific to our decision-making tasks, but may apply to tasks more broadly. Nevertheless, follow-up studies should examine an even broader range of tasks that engage widely different cognitive processes to gain further insight into the functional dynamics of the IFPN and DMN.

A second potential limitation of our study lies in the design of the data collection. Each participant performed the three tasks in the same order, and then completed the resting-state scan last. Prior research suggests that recent exposure to a task may alter resting-state connectivity for several minutes post task (Waites et al., 2005; Stevens et al., 2010; Tung et al., 2013). This concern is partially ameliorated in our study, however, because we compared resting-state data to each of three tasks that differed in their relative timing before the resting-state scan (e.g., by minutes to nearly an hour). Additionally, task-related influences on resting-state connectivity may be minimized or eliminated when comparing differences between task and rest, thus partially precluding task-based changes. Thus, we conclude that recent task performance may have partially contributed to our observed findings; future studies with a balanced order of task and resting scans will be needed to address this issue. Last, because our analyses pertained to the cognitive state, there does remain the possibility that participants experienced a short transition period of rest within each task. However, we believe that even in any potential moments of rest within each task (e.g., during the ITIs), subjects are still anticipating a task and are thus in a qualitatively different cognitive state than during the restingstate scan. Support for this hypothesis comes from nonhuman primate single-unit recordings (Hayden et al., 2009), which found that neuronal activity in the posterior cingulate was significantly different during ITIs compared with a cued rest period. Future imaging work should examine whether patterns of connectivity with the precuneus also reflect these brief changes in cognitive state.

Despite these limitations, our results endorse the idea that the precuneus simultaneously interacts with both the default-mode and frontoparietal networks to distinguish distinct cognitive states. Our results also indicate that the precuneus, a notoriously enigmatic region (Cavanna and Trimble, 2006), serves as a spe- cialized hub within the DMN. Importantly, understanding both the function and specialization within large-scale RSNs may provide insight into disorders marked by disruptions in functional connectivity, particularly schizophrenia (Whitfield-Gabrieli et al., 2009), depression (Greicius et al., 2007), and autism (Kennedy et al., 2006).

\section{References}

Addis DR, McIntosh AR, Moscovitch M, Crawley AP, McAndrews MP (2004) Characterizing spatial and temporal features of autobiographical memory retrieval networks: a partial least squares approach. Neuroimage 23:1460-1471. CrossRef Medline

Beckmann CF, Smith SM (2004) Probabilistic independent component analysis for functional magnetic resonance imaging. IEEE Trans Med Imaging 23:137-152. CrossRef Medline

Beckmann CF, DeLuca M, Devlin JT, Smith SM (2005) Investigations into resting-state connectivity using independent component analysis. Philos Trans R Soc Lond B Biol Sci 360:1001-1013. CrossRef Medline

Buckner RL, Carroll DC (2007) Self-projection and the brain. Trends Cogn Sci 11:49-57. CrossRef Medline

Buckner RL, Sepulcre J, Talukdar T, Krienen FM, Liu H, Hedden T, AndrewsHanna JR, Sperling RA, Johnson KA (2009) Cortical hubs revealed by intrinsic functional connectivity: mapping, assessment of stability, and relation to Alzheimer's disease. J Neurosci 29:1860-1873. CrossRef Medline

Bullmore E, Sporns O (2009) Complex brain networks: graph theoretical analysis of structural and functional systems. Nat Rev Neurosci 10:186198. CrossRef Medline

Button KS, Ioannidis JP, Mokrysz C, Nosek BA, Flint J, Robinson ES, Munafò MR (2013) Power failure: why small sample size undermines the reliability of neuroscience. Nat Rev Neurosci 14:365-376. CrossRef Medline

Cavanna AE, Trimble MR (2006) The precuneus: a review of its functional anatomy and behavioural correlates. Brain 129:564-583. CrossRef Medline

Clithero JA, Reeck C, Carter RM, Smith DV, Huettel SA (2011) Nucleus accumbens mediates relative motivation for rewards in the absence of choice. Front Hum Neurosci 5:87. Medline

Cole DM, Smith SM, Beckmann CF (2010) Advances and pitfalls in the analysis and interpretation of resting-state FMRI data. Front Syst Neurosci 4:8. Medline

Damoiseaux JS, Rombouts SA, Barkhof F, Scheltens P, Stam CJ, Smith SM, Beckmann CF (2006) Consistent resting-state networks across healthy subjects. Proc Natl Acad Sci U S A 103:13848-13853. CrossRef Medline

De Martino B, Kumaran D, Seymour B, Dolan RJ (2006) Frames, biases, and rational decision-making in the human brain. Science 313:684-687. CrossRef Medline

Eustache F, Piolino P, Giffard B, Viader F, De La Sayette V, Baron JC, Desgranges B (2004) "In the course of time": a PET study of the cerebral substrates of autobiographical amnesia in Alzheimer's disease. Brain 127: 1549-1560. CrossRef Medline

Filippi M, Valsasina P, Misci P, Falini A, Comi G, Rocca MA (2013) The organization of intrinsic brain activity differs between genders: a restingstate fMRI study in a large cohort of young healthy subjects. Hum Brain Mapp 34:1330-1343. Medline

Filippini N, MacIntosh BJ, Hough MG, Goodwin GM, Frisoni GB, Smith SM, Matthews PM, Beckmann CF, Mackay CE (2009) Distinct patterns of brain activity in young carriers of the APOE- $\varepsilon 4$ allele. Proc Natl Acad Sci U S A 106:7209-7214. CrossRef Medline

Fletcher PC, Frith CD, Baker SC, Shallice T, Frackowiak RS, Dolan RJ (1995) The mind's eye - precuneus activation in memory-related imagery. Neuroimage 2:195-200. CrossRef Medline

Fox MD, Raichle ME (2007) Spontaneous fluctuations in brain activity observed with functional magnetic resonance imaging. Nat Rev Neurosci 8:700-711. CrossRef Medline

Fox MD, Snyder AZ, Vincent JL, Corbetta M, Van Essen DC, Raichle ME (2005) The human brain is intrinsically organized into dynamic, anticorrelated functional networks. Proc Natl Acad Sci U S A 102:9673-9678. CrossRef Medline

Fox MD, Corbetta M, Snyder AZ, Vincent JL, Raichle ME (2006) Spontaneous neuronal activity distinguishes human dorsal and ventral attention systems. Proc Natl Acad Sci U S A 103:10046-10051. CrossRef Medline 
Fransson P (2005) Spontaneous low-frequency BOLD signal fluctuations: an fMRI investigation of the resting-state default mode of brain function hypothesis. Hum Brain Mapp 26:15-29. CrossRef Medline

Fransson P, Marrelec G (2008) The precuneus/posterior cingulate cortex plays a pivotal role in the default mode network: evidence from a partial correlation network analysis. Neuroimage 42:1178-1184. CrossRef Medline

Friedman L, Glover GH (2006) Reducing interscanner variability of activation in a multicenter fMRI study: controlling for signal-tofluctuation-noise-ratio (SFNR) differences. Neuroimage 33:471-481. CrossRef Medline

Friston KJ (2011) Functional and effective connectivity: a review. Brain Connect 1:13-36. CrossRef Medline

Gordon EM, Stollstorff M, Vaidya CJ (2012) Using spatial multiple regression to identify intrinsic connectivity networks involved in working memory performance. Hum Brain Mapp 33:1536-1552. CrossRef Medline

Greicius MD, Menon V (2004) Default-mode activity during a passive sensory task: uncoupled from deactivation but impacting activation. J Cogn Neurosci 16:1484-1492. CrossRef Medline

Greicius MD, Krasnow B, Reiss AL, Menon V (2003) Functional connectivity in the resting brain: a network analysis of the default mode hypothesis. Proc Natl Acad Sci U S A 100:253-258. CrossRef Medline

Greicius MD, Flores BH, Menon V, Glover GH, Solvason HB, Kenna H, Reiss AL, Schatzberg AF (2007) Resting-state functional connectivity in major depression: abnormally increased contributions from subgenual cingulate cortex and thalamus. Biol Psychiatry 62:429-437. CrossRef Medline

Gusnard DA, Raichle ME (2001) Searching for a baseline: functional imaging and the resting human brain. Nat Rev Neurosci 2:685-694. CrossRef Medline

Hayden BY, Nair AC, McCoy AN, Platt ML (2008) Posterior cingulate cortex mediates outcome-contingent allocation of behavior. Neuron 60:1925. CrossRef Medline

Hayden BY, Smith DV, Platt ML (2009) Electrophysiological correlates of default-mode processing in macaque posterior cingulate cortex. Proc Natl Acad Sci U S A 106:5948-5953. CrossRef Medline

Hayden BY, Smith DV, Platt ML (2010) Cognitive control signals in posterior cingulate cortex. Front Hum Neurosci 4:223. Medline

Huettel SA, Stowe CJ, Gordon EM, Warner BT, Platt ML (2006) Neural signatures of economic preferences for risk and ambiguity. Neuron 49: 765-775. CrossRef Medline

Hyvärinen A (1999) Fast and robust fixed-point algorithms for independent component analysis. IEEE Trans Neural Netw 10:626-634. CrossRef Medline

Jansen M, White TP, Mullinger KJ, Liddle EB, Gowland PA, Francis ST, Bowtell R, Liddle PF (2012) Motion-related artefacts in EEG predict neuronally plausible patterns of activation in fMRI data. Neuroimage 59:261-270. CrossRef Medline

Jenkinson M, Smith S (2001) A global optimisation method for robust affine registration of brain images. Med Image Anal 5:143-156. CrossRef Medline

Kennedy DP, Redcay E, Courchesne E (2006) Failing to deactivate: resting functional abnormalities in autism. Proc Natl Acad Sci U S A 103:82758280. CrossRef Medline

Knutson B, Westdorp A, Kaiser E, Hommer D (2000) FMRI visualization of brain activity during a monetary incentive delay task. Neuroimage 12:2027. CrossRef Medline

Leech R, Sharp DJ (2013) The role of the posterior cingulate cortex in cognition and disease. Brain. Advance online publication. Retrieved September 5, 2013. doi:10.1093/brain/awt162. CrossRef Medline

Leech R, Kamourieh S, Beckmann CF, Sharp DJ (2011) Fractionating the default mode network: distinct contributions of the ventral and dorsal posterior cingulate cortex to cognitive control. J Neurosci 31:3217-3224. CrossRef Medline

Leech R, Braga R, Sharp DJ (2012) Echoes of the brain within the posterior cingulate cortex. J Neurosci 32:215-222. CrossRef Medline

Lundstrom BN, Ingvar M, Petersson KM (2005) The role of precuneus and left inferior frontal cortex during source memory episodic retrieval. Neuroimage 27:824-834. CrossRef Medline
Maddock RJ, Garrett AS, Buonocore MH (2003) Posterior cingulate cortex activation by emotional words: fMRI evidence from a valence decision task. Hum Brain Mapp 18:30-41. CrossRef Medline

Maddock RJ, Garrett A, Buonocore MH (2001) Remembering familiar people: the posterior cingulate cortex and autobiographical memory retrieval. Neuroscience 104:667-676. CrossRef Medline

McKiernan KA, Kaufman JN, Kucera-Thompson J, Binder JR (2003) A parametric manipulation of factors affecting task-induced deactivation in functional neuroimaging. J Cogn Neurosci 15:394-408. CrossRef Medline

Mennes M, Kelly C, Zuo XN, Di Martino A, Biswal BB, Castellanos FX, Milham MP (2010) Inter-individual differences in resting state functional connectivity predict task-induced BOLD activity. Neuroimage 50: 1690-1701. CrossRef Medline

Mennes M, Kelly C, Colcombe S, Castellanos FX, Milham MP (2013) The extrinsic and intrinsic functional architectures of the human brain are not equivalent. Cereb Cortex 23:223-229. CrossRef Medline

Niazy RK, Xie J, Miller K, Beckmann CF, Smith SM (2011) Spectral characteristics of resting state networks. Prog Brain Res 193:259-276. CrossRef Medline

Nichols TE, Holmes AP (2002) Nonparametric permutation tests for functional neuroimaging: a primer with examples. Hum Brain Mapp 15:1-25. CrossRef Medline

Nichols T, Brett M, Andersson J, Wager T, Poline JB (2005) Valid conjunction inference with the minimum statistic. Neuroimage 25:653-660. CrossRef Medline

O'Reilly JX, Woolrich MW, Behrens TE, Smith SM, Johansen-Berg H (2012) Tools of the trade: psychophysiological interactions and functional connectivity. Soc Cogn Affect Neurosci 7:604-609. CrossRef Medline

Pearson JM, Heilbronner SR, Barack DL, Hayden BY, Platt ML (2011) Posterior cingulate cortex: adapting behavior to a changing world. Trends Cogn Sci 15:143-151. CrossRef Medline

Power JD, Barnes KA, Snyder AZ, Schlaggar BL, Petersen SE (2012) Spurious but systematic correlations in functional connectivity MRI networks arise from subject motion. Neuroimage 59:2142-2154. CrossRef Medline

Pruessmann KP, Weiger M, Börnert P, Boesiger P (2001) Advances in sensitivity encoding with arbitrary k-space trajectories. Magn Reson Med 46:638-651. CrossRef Medline

Raichle ME, MacLeod AM, Snyder AZ, Powers WJ, Gusnard DA, Shulman GL (2001) A default mode of brain function. Proc Natl Acad Sci U S A 98:676-682. CrossRef Medline

Satterthwaite TD, Wolf DH, Loughead J, Ruparel K, Elliott MA, Hakonarson H, Gur RC, Gur RE (2012) Impact of in-scanner head motion on multiple measures of functional connectivity: relevance for studies of neurodevelopment in youth. Neuroimage 60:623-632. CrossRef Medline

Shapira-Lichter I, Oren N, Jacob Y, Gruberger M, Hendler T (2013) Portraying the unique contribution of the default mode network to internally driven mnemonic processes. Proc Natl Acad Sci U S A 110:4950-4955. CrossRef Medline

Sharp DJ, Beckmann CF, Greenwood R, Kinnunen KM, Bonnelle V, De Boissezon X, Powell JH, Counsell SJ, Patel MC, Leech R (2011) Default mode network functional and structural connectivity after traumatic brain injury. Brain 134:2233-2247. CrossRef Medline

Shulman GL, Fiez JA, Corbetta M, Buckner RL, Miezin FM, Raichle ME, Petersen SE (1997) Common blood flow changes across visual tasks: II. Decreases in cerebral cortex. J Cogn Neurosci 9:648-663. CrossRef Medline

Sladky R, Friston KJ, Tröstl J, Cunnington R, Moser E, Windischberger C (2011) Slice-timing effects and their correction in functional MRI. Neuroimage 58:588-594. CrossRef Medline

Smith SM (2002) Fast robust automated brain extraction. Hum Brain Mapp 17:143-155. CrossRef Medline

Smith SM (2012) The future of FMRI connectivity. Neuroimage 62:12571266. CrossRef Medline

Smith SM, Nichols TE (2009) Threshold-free cluster enhancement: addressing problems of smoothing, threshold dependence and localisation in cluster inference. Neuroimage 44:83-98. CrossRef Medline

Smith SM, Jenkinson M, Woolrich MW, Beckmann CF, Behrens TE, Johansen-Berg H, Bannister PR, De Luca M, Drobnjak I, Flitney DE, 
Niazy RK, Saunders J, Vickers J, Zhang Y, De Stefano N, Brady JM, Matthews PM (2004) Advances in functional and structural MR image analysis and implementation as FSL. Neuroimage 23:S208-S219. CrossRef Medline

Smith SM, Fox PT, Miller KL, Glahn DC, Fox PM, Mackay CE, Filippini N, Watkins KE, Toro R, Laird AR, Beckmann CF (2009) Correspondence of the brain's functional architecture during activation and rest. Proc Natl Acad Sci U S A 106:13040-13045. CrossRef Medline

Sporns O, Honey CJ, Kötter R (2007) Identification and classification of hubs in brain networks. PLoS One 2:e1049. CrossRef Medline

Stevens WD, Buckner RL, Schacter DL (2010) Correlated low-frequency BOLD fluctuations in the resting human brain are modulated by recent experience in category-preferential visual regions. Cereb Cortex 20:19972006. CrossRef Medline

Tomasi D, Volkow ND (2010) Functional connectivity density mapping. Proc Natl Acad Sci U S A 107:9885-9890. CrossRef Medline

Truong TK, Song AW (2008) Single-shot dual-z-shimmed sensitivity- encoded spiral-in/out imaging for functional MRI with reduced susceptibility artifacts. Magn Reson Med 59:221-227. CrossRef Medline

Tung KC, Uh J, Mao D, Xu F, Xiao G, Lu H (2013) Alterations in resting functional connectivity due to recent motor task. Neuroimage 78:316324. CrossRef Medline

Waites AB, Stanislavsky A, Abbott DF, Jackson GD (2005) Effect of prior cognitive state on resting state networks measured with functional connectivity. Hum Brain Mapp 24:59-68. CrossRef Medline

Whitfield-Gabrieli S, Thermenos HW, Milanovic S, Tsuang MT, Faraone SV, McCarley RW, Shenton ME, Green AI, Nieto-Castanon A, LaViolette P, Wojcik J, Gabrieli JD, Seidman LJ (2009) Hyperactivity and hyperconnectivity of the default network in schizophrenia and in firstdegree relatives of persons with schizophrenia. Proc Natl Acad Sci U S A 106:1279-1284. CrossRef Medline

Woolrich MW, Jbabdi S, Patenaude B, Chappell M, Makni S, Behrens T, Beckmann C, Jenkinson M, Smith SM (2009) Bayesian analysis of neuroimaging data in FSL. Neuroimage 45:S173-86. CrossRef Medline 